\title{
USP22 promotes epithelial-mesenchymal transition via the FAK pathway in pancreatic cancer cells
}

\author{
ZHEN NING $^{1 *}$, AMAN WANG $^{2 *}$, JINXIAO LIANG $^{1}$, YUNPENG XIE $^{3}$, \\ JIWEI LIU ${ }^{2}$, QIU YAN ${ }^{3}$ and ZHONGYU WANG ${ }^{1}$
}

\begin{abstract}
Departments of ${ }^{1}$ General Surgery and ${ }^{2}$ Oncology, The First Affiliated Hospital, Dalian Medical University, Dalian, Liaoning;
${ }^{3}$ Department of Biochemistry and Molecular Biology, Dalian Medical University, Liaoning Provincial Core Laboratory of Glycobiology and Glycoengineering, Dalian, Liaoning, P.R. China
\end{abstract}

Received March 22, 2014; Accepted June 27, 2014

DOI: $10.3892 /$ or.2014.3354

\begin{abstract}
Epithelial-mesenchymal transition (EMT) contributes to the occurrence and development of tumors, particularly to the promotion of tumor invasion and metastasis. As a newly discovered ubiquitin hydrolase family member, USP22 plays a key role in the malignant transformation of tumors and the regulation of the cell cycle. However, recent studies on USP22 have primarily focused on its role in cell cycle regulation, and the potential mechanism underlying the promotion of tumor invasion and metastasis by abnormal USP22 expression has not been reported. Our studies revealed that the overexpression of USP22 in PANC-1 cells promoted Ezrin redistribution and phosphorylation and cytoskeletal remodeling, upregulated expression of the transcription factors Snail and ZEB1 to promote EMT, and increased cellular invasion and migration. In contrast, blockade of USP22 expression resulted in the opposite effects. In addition, the focal adhesion kinase (FAK) signaling pathway was shown to play a key role in the process of EMT induction in PANC-1 cells by USP22. Thus, the present study suggests that USP22 acts as a regulatory protein for EMT in pancreatic cancer, which may provide a new approach for the targeted therapy of pancreatic cancer.
\end{abstract}

\section{Introduction}

The invasion and metastasis of malignant tumors are the primary factors leading to treatment failure and poor prognosis or death in cancer patients. Recent studies have shown that epithelial-mesenchymal transition (EMT) is closely associated with the invasion and metastasis of malignant tumors (1). EMT is a complex molecular program in which

Correspondence to: Dr Zhongyu Wang, Department of General Surgery, The First Affiliated Hospital of Dalian Medical University, No. 222, Zhongshan Road, Dalian, Liaoning 116011, P.R. China E-mail: wangzhongyudl@126.com

*Contributed equally

Key words: epithelial-mesenchymal transition, USP22, focal adhesion kinase, Ezrin, pancreatic cancer epithelial cells lose their polarity and acquire mesenchymal characteristics through cytoskeletal remodeling. Also during this process, the cellular phenotype changes, with the lack of E-cadherin expression serving as the most important marker of EMT (2). The occurrence of EMT enables tumor cells to acquire the ability to infiltrate surrounding tissues and invade adjacent vasculature; as a result, these cells can metastasize to other tissues or organs to form metastatic foci (3). Indeed, it has been confirmed that EMT can promote the invasion and metastasis of many solid tumors including bladder, liver, colorectal, ovarian, esophageal, breast and prostate cancer (4).

Pancreatic cancer is the fourth leading cause of tumorrelated death in the USA and is associated with a high degree of malignancy and poor prognosis (5). Lymphatic, vascular and distant organ metastases can occur at an early stage; therefore, when pancreatic cancer is confirmed, most patients are at intermediate or advanced stages and have passed the optimal time for surgical treatment (6).

Currently, it is believed that EMT is closely associated with invasion, metastasis and drug resistance in pancreatic cancer (7). Among the pancreatic cancer cell lines examined in previous studies, $78 \%$ expressed Snail, 50\% expressed Slug, and Twist was activated under hypoxic stimulation (8). Studies have further shown that in PANC-1 cells, treatment with transforming growth factor (TGF) promotes EMT through the promotion of $\mathrm{N}$-cadherin and vimentin expression and the inhibition of E-cadherin expression (9). Furthermore, immunohistochemical analysis of a large number of histopathological sections of pancreatic cancer showed that EMT occurrence was significantly correlated with poor prognosis in pancreatic cancer (10). In addition, the characteristics of the EMT phenotype were also observed in gemcitabine-resistant pancreatic cancer cells (11). These results indicate that EMT not only participates in the progression of pancreatic cancer but is also closely associated with drug resistance in pancreatic cancer.

USP22 is a ubiquitin-specific peptidase that belongs to the deubiquitinating enzyme (DUB) family and serves as a subunit of the hSAGA complex (12). USP22 deubiquitinates the $\mathrm{H} 2 \mathrm{~A}$ and $\mathrm{H} 2 \mathrm{~B}$ histone proteins and acetylates the $\mathrm{H} 4$ histone protein (13). In addition, USP22 can activate BMI-1-, c-Myc- and FBP-1-mediated target gene transcription and 
plays a key role in cell cycle regulation, embryonic development and telomere homeostasis (14-17). USP22 expression in normal tissues is low, but its expression is significantly higher in tumors (12). Recently, it has been shown that USP22 is closely associated with the metastatic potential and prognosis of many solid tumors. However, the mechanism underlying the role of abnormal USP22 expression in the occurrence and development of tumors and its regulatory aspects remain poorly defined; moreover, the association between USP22 and pancreatic cancer has not yet been reported.

The present study showed that USP22 is closely associated with EMT occurrence in PANC-1 cells. In particular, upregulation of USP22 expression promoted the redistribution and phosphorylation of Ezrin protein through the focal adhesion kinase (FAK) signaling pathway, which led to cytoskeletal remodeling, the promotion of EMT, upregulated MMP2/ MMP9 expression, and the increased invasion and migration of PANC-1 cells. In contrast, interference with USP22 expression resulted in the opposite effects. Overall, our results suggest that USP22 represents a novel regulatory protein of EMT in pancreatic cancer, which may provide a new approach for the targeted therapy of pancreatic cancer.

\section{Materials and methods}

Cell culture. PANC-1 and AsPC-1 cells were cultured in DMEM/F12 medium (Gibco, Grand Island, NY, USA) supplemented with $10 \%$ fetal bovine serum (FBS) (Invitrogen, Karlsruhe, Germany); CFPAC-1 cells were maintained in IMDM medium supplemented with $10 \%$ FBS. BxPC-3 cells were maintained in RPMI-1640 (both from Gibco) supplemented with $10 \%$ FBS. All cells were cultured in cell-culture flasks or Petri dishes in a humidified incubator at $37^{\circ} \mathrm{C}$ in an atmosphere of $5 \% \mathrm{CO}_{2}$.

Plasmid preparation and cell transfection. The USP22 and FAK overexpression plasmids and USP22-shRNA plasmids and FAK-siRNA were designed and synthesized by Shanghai GenePharma Co.,Ltd. (Shanghai, China). All transfection reactions were performed using Lipofectamine 2000 (Invitrogen, Carlsbad, CA, USA) in accordance with the manufacturer's instructions. Stable transfectants were selected with $800 \mu \mathrm{g} / \mathrm{ml}$ G418 (Sigma-Aldrich, St. Louis, MO, USA), and individual clones were isolated.

Western blotting. Proteins were separated by SDS-PAGE and transferred to nitrocellulose membranes (Bio-Rad, Hercules, CA, USA). Membranes were blocked in a buffer (TBS: $50 \mathrm{mM}$ Tris- $\mathrm{HCl}, 150 \mathrm{mM} \mathrm{NaCl}, \mathrm{pH}$ 7.4) containing 5\% bovine serum albumin and $0.1 \%$ Tween-20, followed by incubation with the primary antibodies. The immunoreactive proteins were visualized using the ECL Western Blotting System (Bio-Rad), and densitometric analysis was performed using BioImaging systems (LabWorks ${ }^{\mathrm{TM}}$, version 4.6; UVP). Mean values of the data obtained from three separate chambers are presented.

Immunofluorescence. PANC-1 cells were seeded onto coverslips, fixed with $4 \%$ paraformaldehyde and permeabilized with $0.3 \%$ Triton X-100 for 10 min. Slides were blocked with $1 \%$ bovine serum albumin and incubated with the primary antibodies overnight at $4^{\circ} \mathrm{C}$. After washing in PBS, the cells were stained with secondary antibodies and incubated for $1 \mathrm{~h}$ at room temperature, followed by nuclear counterstaining with DAPI. Images were captured with the 3i Marianas XL spinning disk confocal microscope (SDCM).

In vitro wound-healing migration assay. Cells were seeded in 6-well culture plates in DMEM/F12 containing 10\% FBS. After $24 \mathrm{~h}$, the cell monolayers were wounded by manually scratching them with a pipette tip, and this was followed by washing with PBS. The monolayers were then incubated at $37^{\circ} \mathrm{C}$ for $24 \mathrm{~h}$. The monolayers were photographed at 0 and $24 \mathrm{~h}$. Images were captured with the Olympus BX51 fluorescence microscope. Mean values of the data obtained from three separate chambers are presented.

Transfilter invasion assay. Transfilter assays were performed with $8.0-\mu \mathrm{m}$ pore inserts in 24-well BioCoat chambers (Becton-Dickinson) using 5x10 4 cells in serum-free Dulbecco's modified Eagle's medium (DMEM). The DMEM with $10 \%$ FBS was placed in the lower chambers as a chemoattractant. For the invasion assays, Matrigel-coated Transwell chambers were used. Cells were removed from the upper surface of the filter by scraping with a cotton swab after $24 \mathrm{~h}$ in culture, respectively. Invasive cells were fixed and stained with the crystal violet reagent. Images were captured with the Olympus BX51 fluorescence microscope. Mean values of the data obtained from three separate chambers are presented.

Statistical analysis. The quantitative data derived from three independent experiments are expressed as means ( \pm SD). Unpaired Student's t-tests were used to analyze between group differences that is repeated. $\mathrm{P}<0.05$ was considered to indicate a statistically significant result.

\section{Results}

Establishment of USP22 overexpression monoclones and silencing of the USP22 gene in PANC-1 cells. We first detected USP22 protein expression in 4 different pancreatic cancer cell lines that were stored in our laboratory. The results showed that USP22 expression in poorly differentiated cell lines, including BxPC-3, AsPC-1 and PANC-1, was significantly higher than that observed in highly differentiated CFPAC-1 cells (Fig. 1A). In addition, USP22 expression in the PANC-1 cells was significantly lower than that detected in the BxPC-3 and AsPC-1 cells. Therefore, the PANC-1 cell line demonstrated a moderate expression level and was selected for use in subsequent experiments.

To study the function of USP22 in pancreatic cancer cells, a USP22 overexpression plasmid and an shRNA were stably transfected into PANC-1 cells. After G418 selection, the cell clones Panc-usp and Panc-uspsh, which were stably transfected with the corresponding plasmids, were selected for future studies. Western blot results showed that the expression levels of USP22 in the Panc-usp and Panc-uspsh cells were 2.11-fold greater and $30.6 \%$ of the level observed in the PANC-1 cells, respectively (Fig. 1B). In addition, the expression level of USP22 in the Panc-usp cells was 5.3-fold greater than that detected in the Panc-uspsh cells. 

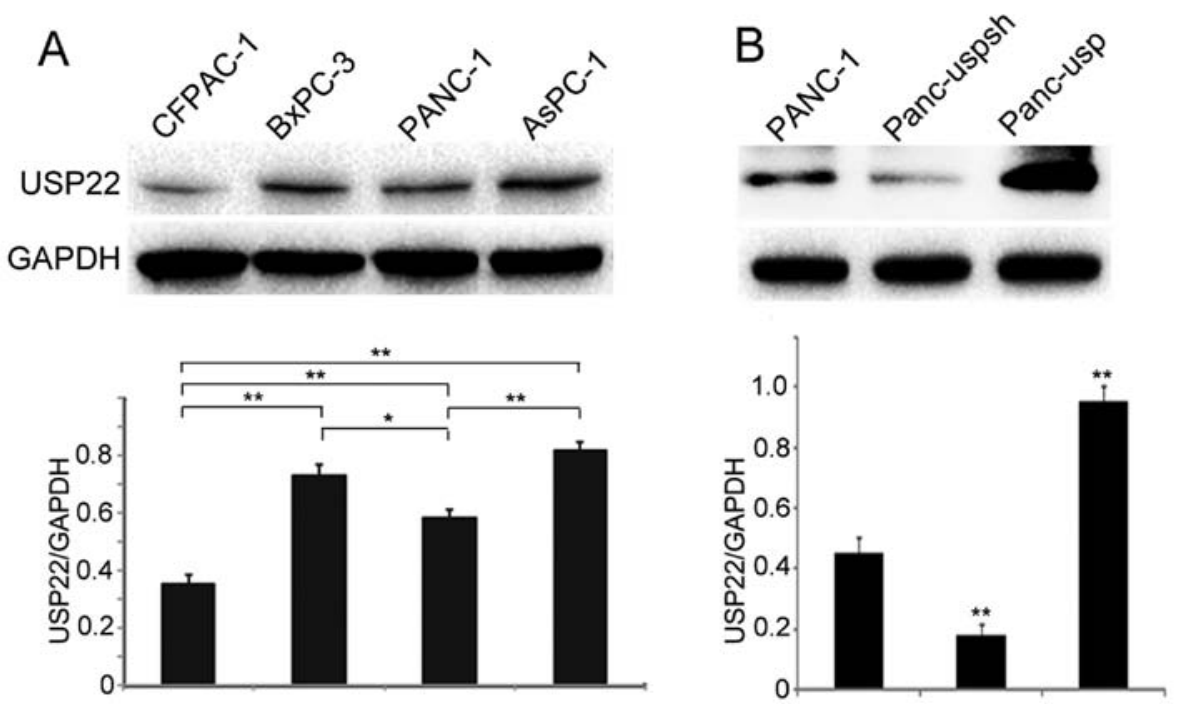

Figure 1. Stable overexpression and silencing of USP22 in PANC-1 cells. (A) Western blot analysis of USP22 expression in BxPC-3, AsPC-1, PANC-1 and CFPAC-1 pancreatic cancer cell lines. (B) Western blot analysis of USP22 expression in PANC-1, Panc-usp (cell clones which were stably transfected with the USP22 overexpression plasmids) and Panc-uspsh (cell clones which were stably transfected with the USP22-shRNA) cells.

USP22 promotes the redistribution and phosphorylation of Ezrin and cytoskeletal remodeling in PANC-1 cells. Cytoskeletal remodeling is a key step in EMT. To examine whether USP22 promotes EMT in PANC-1 cells, detection of F-actin expression in Panc-usp and Panc-uspsh cells was performed using laser-scanning confocal immunofluorescence. The results showed that there was a large amount of longitudinally oriented actin filaments in the cytoplasm of the Panc-usp cells, whereas in the Panc-uspsh cells, actin showed punctate expression without actin filaments in the cytoplasm (Fig. 2B). Next, the expression of Ezrin protein, an important regulatory and cell adhesion protein for connections to the cell membrane (CD44 and intercellular adhesion molecule-2) and the cytoskeleton (actin), was determined. The results showed that Ezrin expression in the Panc-uspsh cells was mainly concentrated in the cytoplasm (Fig. 2A). Interestingly, in USP22 stably expressing Panc-usp cells, Ezrin expression was significantly closer to the cell membrane and almost absent in the cytoplasm. The subsequent western blot results showed that although the expression levels of Ezrin in the PANC-1, Panc-uspsh and Panc-usp cells did not change, the levels of Ezrin phosphorylation (P-Ezrin) were significantly altered (Fig. 2C). For example, the expression level of P-Ezrin in the Panc-usp cells was 6.4-fold greater than that observed in the Panc-uspsh cells. These results indicate that USP22 significantly affects cytoskeletal remodeling in pancreatic cancer cells, which may be associated with the redistribution and phosphorylation of Ezrin.

USP22 promotes Ezrin phosphorylation through the FAK signaling pathway. To clarify the potential mechanism underlying the promotion of Ezrin phosphorylation by USP22 overexpression, the FAK signaling pathway, which is loosely associated with invasion, metastasis and adhesion of tumor cells, was studied. Western blot results showed that the expression level of P-FAK in the Panc-usp cells was 3.6-fold greater than that in the Panc-uspsh cells. To further confirm the function of the FAK signaling pathway, control-siRNA and FAK-siRNA were separately transfected into the Panc-usp cells. The results showed that with the decrease in FAK expression, P-PAK and P-Ezrin expression was also significantly decreased (Fig. 2E). Furthermore, transfection of an empty plasmid or an FAK overexpression plasmid into the Panc-uspsh cells showed that with the increase in FAK expression, P-FAK and P-Ezrin expression also increased (Fig. 2D). These results showed that USP22 promoted Ezrin protein phosphorylation through activation of the FAK signaling pathway.

Overexpression of USP22 induces phenotypic changes and lead to the acquisition of mesenchymal markers and reduction of epithelial markers in PANC-1 cells. To clarify whether USP22 promotes EMT, the expression of EMT-associated molecular markers and transcription factors, including ZEB1 and Snail, in PANC-1 cells was evaluated. Western blot results for Panc-usp cells showed that the expression levels of the mesenchymal markers $\mathrm{N}$-cadherin, vimentin and fibronectin were increased 5.8-, 4.2- and 2.7-fold compared to those in Panc-uspsh cells (Fig. 3A), while the expression level of the epithelial marker E-cadherin was decreased by $75.3 \%$ compared to that in Panc-uspsh cells. The evaluation of EMT-associated transcription factors showed that the expression levels of ZEB1 and Snail in the Panc-usp cells were 5.6- and 5.3-fold greater than those in the Panc-uspsh cells.

The expression of E-cadherin protein was also determined using laser-scanning confocal immunofluorescence. The results showed that USP22 stably downregulated Panc-uspsh cells were closely connected and showed a large amount of E-cadherin aggregated at the cell membrane. In contrast, USP22 stably overexpressing Panc-usp cells were loosely connected, extended and spindle-shaped, and E-cadherin protein expression was significantly downregulated and scattered throughout the cytoplasm (Fig. 2A). Thus, these results strongly suggest that USP22 induces EMT in PANC-1 cells through the upregulation of ZEB1 and Snail, accompanied by 

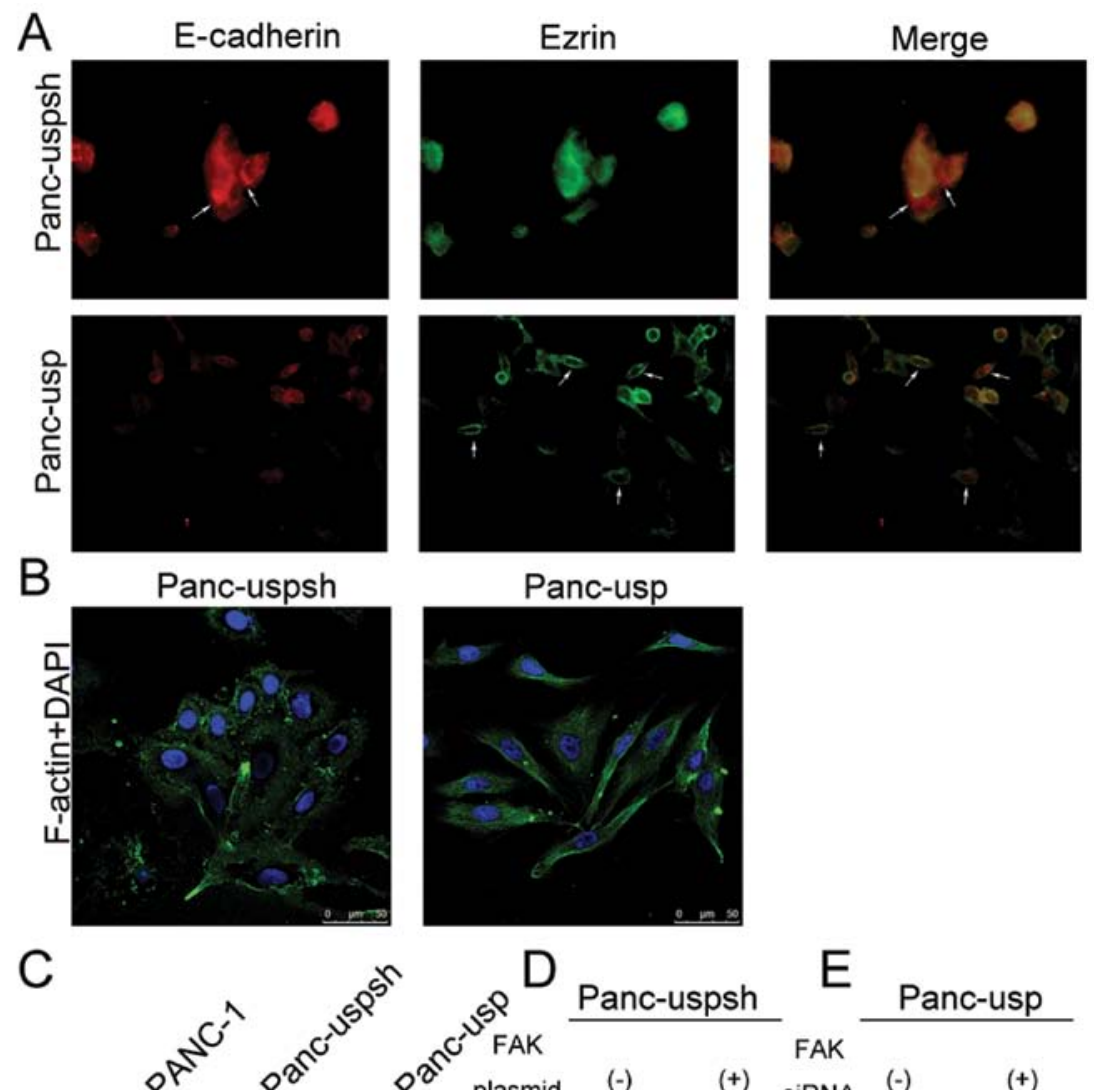

E
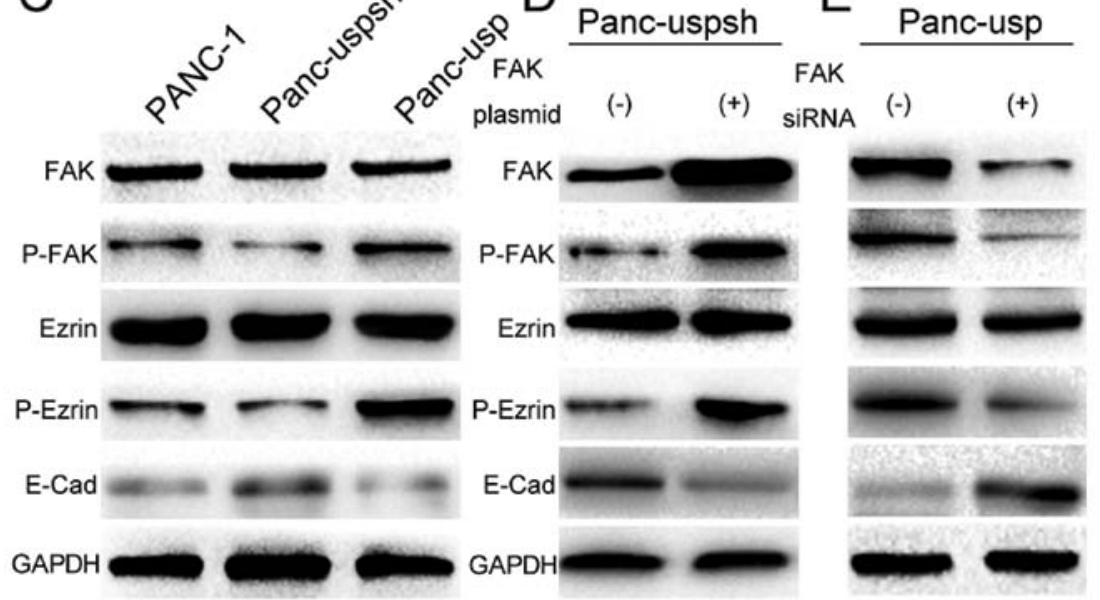

Figure 2. USP22 promotes the redistribution and phosphorylation of Ezrin and cytoskeletal remodeling in PANC-1 cells. (A) Immunofluorescence (IF) staining for E-cadherin (red) and Ezrin (green) was performed in Panc-usp and Panc-uspsh cells. (B) IF staining for F-actin (green) and DAPI (bule) was performed in Panc-usp and Panc-uspsh cells. (C) Western blot analysis of FAK, P-FAK, Ezrin, P-Ezrin and E-cadherin expression in PANC-1, Panc-usp and Panc-uspsh cells. (D and E) Western blot analysis of FAK, P-FAK, Ezrin, P-Ezrin and E-cadherin expression in (E) Panc-usp cells transfected with controlsiRNA or FAK-siRNA and (D) Panc-uspsh cells transfected with vector or FAK plasmids. FAK, focal adhesion kinase.

loss of the EMT marker E-cadherin and gain of the mesenchymal markers vimentin and fibronectin.

USP22 alters the expression of various EMT markers in association with upregulation of the transcription factors ZEBI and Snail via the FAK pathway. To further confirm the role of the FAK signaling pathway in USP22 overexpression-induced EMT, FAK-siRNA was transfected into Panc-usp cells. The results showed that with the downregulation of P-FAK, the expression levels of the mesenchymal markers $\mathrm{N}$-cadherin, vimentin and fibronectin were decreased by $63.8,81.3$ and $64.5 \%$, respectively (Fig. 3C), while the expression level of the epithelial marker E-cadherin was increased 4.2-fold. With the downregulation of P-FAK, the expression levels of the EMT-associated transcription factors ZEB1 and Snail were decreased to 86.2 and $78.4 \%$, respectively. Next, Panc-uspsh cells were transfected with the FAK-overexpressing plasmid, and with the upregulation of P-FAK, the expression levels of the mesenchymal markers $\mathrm{N}$-cadherin, vimentin and fibronectin were increased 5.2-, 1.4- and 3.6-fold, respectively (Fig. 3B), while the expression level of the epithelial marker E-cadherin was decreased by $87.5 \%$. In addition, the expression levels of the EMT-associated transcription factors ZEB1 and Snail were increased 2.8- and 3.6-fold, respectively. These results further indicate that the FAK signaling pathway participates in the USP22 overexpression-induced EMT in PANC-1 cells.

USP22 promotes $P A N C-1$ cell invasion and migration through the FAK signaling pathway. As a result of EMT, the biological behaviors of cells are altered, and cells acquire increased 


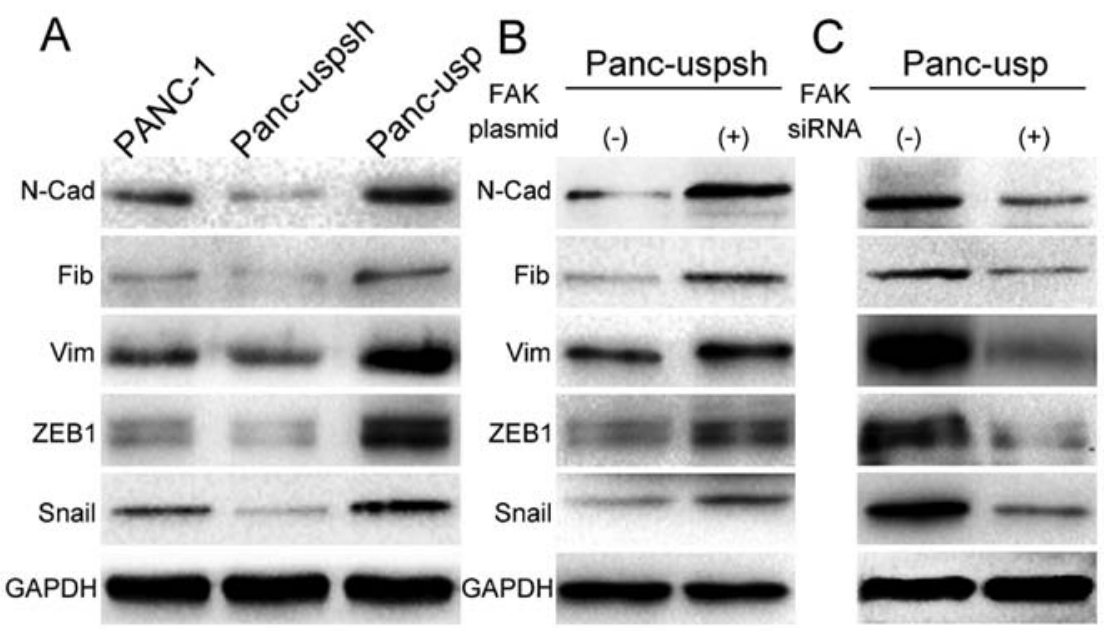

Figure 3. USP22 alters the expression of various EMT markers in association with upregulation of the transcription factors ZEB1 and Snail via the FAK pathway. (A) Western blot analysis of N-cadherin, vimentin, fibronectin, ZEB1 and Snail expression in PANC-1, Panc-usp and Panc-uspsh cells. (B and C) Western blot analysis of N-cadherin, vimentin, fibronectin, ZEB1 and Snail expression in (C) Panc-usp cells transfected with control-siRNA or FAK-siRNA and (B) Panc-uspsh cells transfected with vector or FAK plasmids. EMT, epithelial-mesenchymal transition; FAK, focal adhesion kinase.

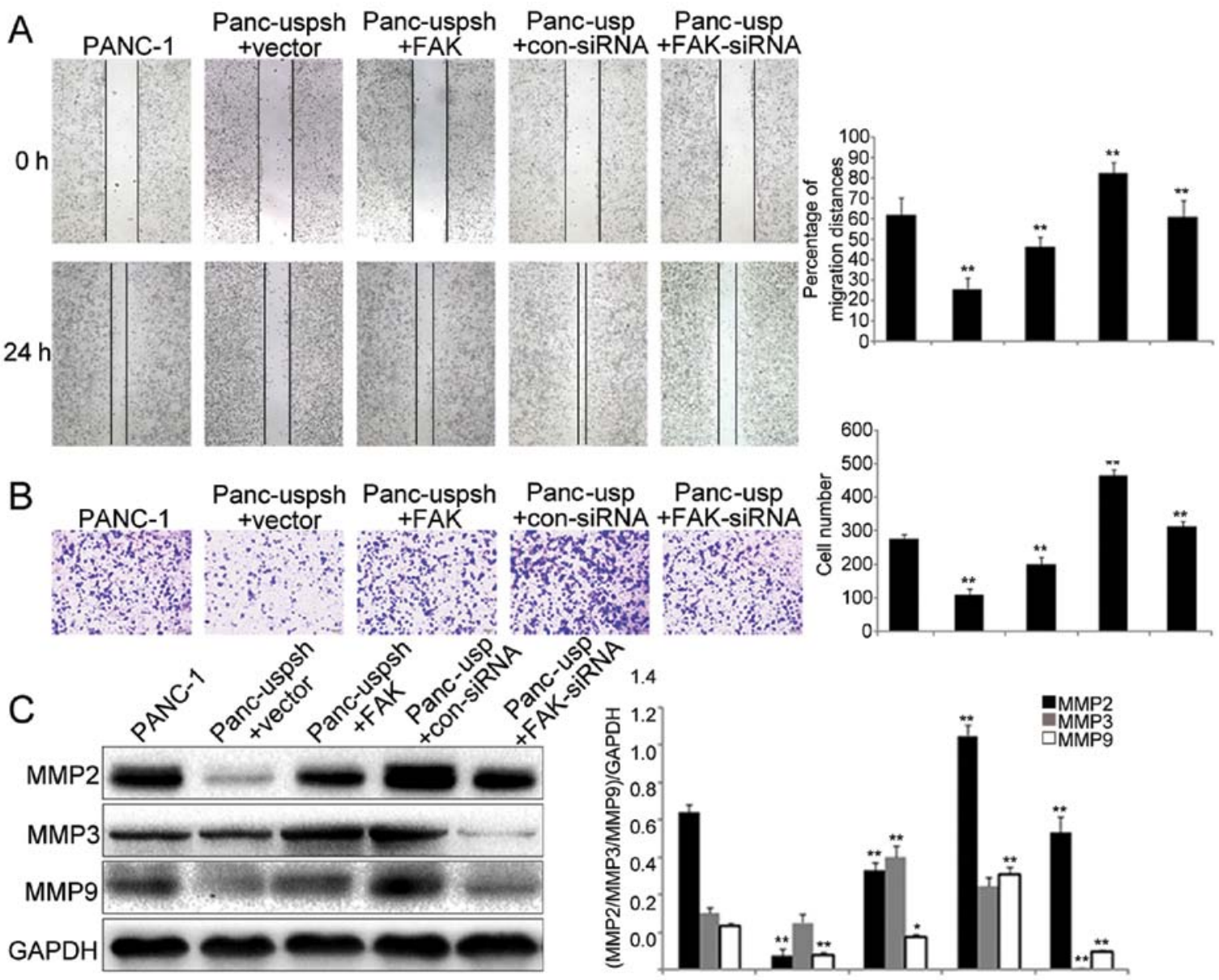

Figure 4. USP22 promotes PANC-1 cell invasion and migration through the FAK signaling pathway. (A) A wound-healing assay was performed in PANC-1 and Panc-usp cells transfected with control-siRNA or FAK-siRNA and Panc-uspsh cells transfected with vector or FAK plasmids. Phase-contrast images were captured at 0 and $24 \mathrm{~h}$ to assess cell migration into the open space. (B) Matrigel-coated Transwell chambers were used to detect cell invasion in PANC-1 cells and Panc-usp cells transfected with control-siRNA or FAK-siRNA and Panc-uspsh cells transfected with vector or FAK plasmids. Representative fields were photographed. (C) Western blot analysis of MMP2, MMP3 and MMP9 expression in PANC-1 cells and in Panc-usp cells transfected with control-siRNA or FAK-siRNA and in Panc-uspsh cells transfected with vector or FAK plasmids. FAK, focal adhesion kinase.

migration and invasion abilities. Using scratch and Transwell assays, we showed that the migration and invasion abilities of the Panc-usp cells were significantly greater than those of the Panc-uspsh cells (Fig. 4A and B). In addition, downregula- 
tion of FAK in the Panc-usp cells significantly decreased the migration and invasion rates, while overexpression of FAK in the Panc-uspsh cells significantly increased the migration and invasion rates.

The expression of MMP2, MMP3 and MMP9 has been shown to play an important role in the process of EMT in various tumors. Our study showed that the expression levels of MMP2 and MMP9 in the Panc-usp cells were significantly increased (by 9.6 and 5.2-fold, respectively) compared to those in the Panc-uspsh cells, while the expression of MP3 was not significantly different (Fig. 4C). In addition, downregulation of FAK in the Panc-usp cells led to significantly downregulated expression of MMP2, MMP3 and MMP9, while overexpression of FAK in the Panc-uspsh cells led to significantly upregulated expression of MMP2, MMP3 and MMP9. These results demonstrated that although the FAK signaling pathway regulated the expression of these three matrix metalloproteinases (MMP2, MMP3 and MMP9), the EMT process in PANC-1 cells induced by USP22 was associated with the expression of MMP2 and MMP9, but not that of MMP3.

\section{Discussion}

EMT was first discovered during the study of embryonic development (18). In-depth studies in human tumors and experimental animal models further revealed that EMT was not only associated with the normal process of embryonic development but was also closely associated with organic fibrosis and tumor invasion and metastasis (3). Yang and Weinberg (19) summarized the following characteristics of changes associated with EMT: i) morphological changes, including loss of cell polarity, spindle-like cell morphology, cytoskeletal remodeling, and appearance of pseudopods; ii) molecular marker changes, including the upregulation of epithelial adhesion-associated molecules such as E-cadherin and downregulation of molecules associated with mesenchymal origin such as $\mathrm{N}$-cadherin and fibronectin; iii) changes in biological behaviors, including the acquisition of mesenchymal phenotypes such as increased migration and invasion and resistance to apoptosis. As one of the protein components of the SAGA complex, USP22 participates in proto-oncogene c-Myc-mediated target gene transcriptional regulation, thus playing a key role in the promotion of malignant transformation of tumors and the regulation of the cell cycle $(16,20)$. Therefore, USP22 is considered to be a tumor stem cell marker. Furthermore, USP22 is also involved in the early developmental process of mouse embryos (21). As a tumor marker, USP22 has recently been shown to be highly expressed in many solid tumors and is closely associated with metastatic potential and poor prognosis. However, recent studies on USP22 have mainly focused on its role in cell cycle regulation, while the potential mechanism underlying the promotion of tumor invasion and metastasis by abnormal USP22 expression has not been reported.

During the process of EMT, epithelial cells downregulate their adhesive structures between cells, change their polarity, and remodel their cytoskeleton to become isolated and mobile anti-apoptotic cells. After EMT is complete, epithelial cells lose their polarity, and the morphology of these cells change from a cubic shape to a spindle-shaped fibroblast-like morphology. As the first discovered ezrin/radixin/moesin (ERM) protein family member, Ezrin is mainly distributed and concentrated at protruding parts of the cell surface such as microvilli and cell folds (22). Ezrin is considered an important regulatory protein and adhesion protein between cell membrane molecules (CD44 and intercellular adhesion molecule-2) and the cellular cytoskeleton (actin), and this protein plays an important role in cytoskeletal remodeling (23). Ezrin is also a regulatory factor for cell motility; studies have shown that stimulation of epithelial tumor cells with hepatocyte growth factor/scatter factor could promote the tyrosine phosphorylation of Ezrin, the movement of Ezrin from the cytoplasm to cell microfolds, and the enhancement of tumor cell mobility (24). The present study showed that USP22 overexpression resulted in the appearance of a large amount of longitudinally oriented actin filaments in the cytoplasm of PANC-1 cells; in contrast, USP22 stably downregulated Panc-uspsh cells exhibited punctate actin expression and the absence of actin filament in the cytoplasm. At the same time, with the increase in USP22 expression, Ezrin expression moved from the cytoplasm to the cell membrane. Moreover, our dynamic observations of cell morphology showed that PANC-1 cells with stable downregulation of USP22 were closely connected and polygonal in shape. In contrast, USP22 stably overexpressing Panc-usp cells were loosely connected and extended and spindle-shaped. These results helped to confirm that USP22 significantly downregulated adhesion between PANC-1 cells, remodeling of the cytoskeleton, and generated cells with an isolated, spindleshaped, fibroblast-like morphology. Moreover, these changes may be associated with the redistribution and phosphorylation of Ezrin.

The most important process in the early stage of EMT is the conversion between E-cadherin and $\mathrm{N}$-cadherin, which is referred to as cadherin conversion. In addition, transcription factors such as Snail1 (Snail), Snail2 (Slug), Snail3, ZEB (including ZEB1 and ZEB2) and Twist can bind to the common box sequences of the promoter region of the E-cadherin gene, thereby downregulating E-cadherin expression (25). Moreover, activated Ezrin protein at the cell membrane can block the translocation of E-cadherin from the cytoplasm to the cell membrane, which indirectly decreases the connections between tumor cells; as a result, tumor cells can more easily leave the primary tumor focus to cause distant metastasis (26). The present study showed that the upregulation of USP22 in PANC-1 cells significantly downregulated E-cadherin expression in the cell membrane at cell-cell junctions and upregulated the expression of mesenchymal markers, including $\mathrm{N}$-cadherin, vimentin and fibronectin. In addition, the expression levels of the transcription factors ZEB1 and Snail were also significantly increased. Together, these results revealed that USP22 promotes the transition of PANC-1 cells from the epithelial phenotype to the mesenchymal phenotype, and this process may be associated with the activation of Ezrin.

The final step in EMT is the acquisition of migration and invasion capacity. Many studies have reported that in gastrointestinal cancers, EMT promotes the occurrence and metastasis of esophageal, gastric, colorectal and pancreatic cancer and is associated with poor prognosis. Matrix metalloproteinases constitute a large family of enzymes that can degrade almost all protein components in the extracellular matrix (ECM), thereby removing a barrier for tumor cell inva- 
sion consequently promoting metastasis (27). Among these enzymes, the type IV collagenases represent an important group that includes two major forms: glycosylated MMP9 and non-glycosylated MMP2. Recent studies have shown that the expression of MMP2, MMP3 and MMP9 plays an important role in the EMT process in many types of tumors $(28,29)$. Using scratch and Transwell assays, the present study found that USP22 overexpression significantly promoted the invasion and migration abilities of PANC-1 cells. In particular, the expression of MMP2 and MMP9, but not MMP3, was associated with USP22-induced EMT.

FAK is a non-receptor type protein tyrosine kinase (30). Previous studies have shown that FAK participates in biological processes such as cell proliferation, adhesion, invasion and apoptosis through its effects on many signal transduction pathways. In particular, FAK plays an important role in regulation of the cytoskeleton and cell motility; through regulating the activation of Rho family proteins, FAK can regulate cell proliferation, adhesion and directional movement (31). Furthermore, Ezrin activation can result in Rho activation and lead to cascading effects in corresponding Rho signal transduction systems (32). These results led us to speculate that FAK may also play an important role in USP22-induced EMT in PANC-1 cells, and the following findings confirmed this speculation. First, P-PAK expression in the USP22 stably expressing Panc-usp cells was significantly higher than that in the USP22-downregulated Panc-uspsh cells. Second, FAK downregulation in the Panc-usp cells significantly reduced the expression of mesenchymal markers, whereas FAK overexpression in the Panc-uspsh cells significantly upregulated the expression of mesenchymal markers. Third, FAK downregulation in the Panc-usp cells significantly downregulated migration and invasion, whereas FAK overexpression in the Panc-uspsh cells significantly upregulated migration and invasion. These results indicate that future research should address the pathway through which USP22 promotes FAK phosphorylation during EMT in PANC-1 cells as well as the relationship between FAK phosphorylation and the redistribution and phosphorylation of Ezrin.

In summary, the present study revealed that USP22 mediated the occurrence of EMT in PANC-1 cells. This process was achieved through activation of the FAK signaling pathway, which promoted the redistribution and phosphorylation of Ezrin protein, cytoskeletal remodeling, EMT occurrence, upregulation of the expression of MMP2/MMP9 and increased invasion and migration in PANC-1 cells. Therefore, downregulation of USP22 may represent an effective treatment method for inhibiting the invasion and migration of pancreatic cancer.

\section{Acknowledgements}

This study was supported by the National Natural Science Foundation of China Research grant (no. 30870719 to Z.W., no. 30672753 to J.L.) and China 973 grant (no. 2012CB822100 to Q.Y.).

\section{References}

1. Thiery JP, Acloque H, Huang RY and Nieto MA: Epithelialmesenchymal transitions in development and disease. Cell 139: 871-890, 2009
2. Thiery JP: Epithelial-mesenchymal transitions in tumour progression. Nat Rev Cancer 2: 442-454, 2002.

3. Brabletz T: EMT and MET in metastasis: where are the cancer stem cells? Cancer Cell 22: 699-701, 2012.

4. Natalwala A, Spychal R and Tselepis C: Epithelial-mesenchymal transition mediated tumourigenesis in the gastrointestinal tract. World J Gastroenterol 14: 3792-3797, 2008.

5. Jemal A, Bray F, Center MM, Ferlay J, Ward E and Forman D: Global cancer statistics. CA Cancer J Clin 61: 69-90, 2011.

6. Hidalgo M: Pancreatic cancer. N Engl J Med 362: 1605-1617, 2010.

7. Pan JJ and Yang MH: The role of epithelial-mesenchymal transition in pancreatic cancer. J Gastrointest Oncol 2: 151-156, 2011.

8. Hotz B, Arndt M, Dullat S, Bhargava S, Buhr HJ and Hotz HG: Epithelial to mesenchymal transition: expression of the regulators snail, slug, and twist in pancreatic cancer. Clin Cancer Res 13: 4769-4776, 2007.

9. Takano S, Kanai F, Jazag A, Ijichi H, Yao J, Ogawa H, Enomoto N, Omata $\mathrm{M}$ and Nakao A: Smad4 is essential for down-regulation of E-cadherin induced by TGF- $\beta$ in pancreatic cancer cell line PANC-1. J Biochem 141: 345-351, 2007.

10. Krantz SB, Shields MA, Dangi-Garimella S, Bentrem DJ and Munshi HG: Contribution of epithelial-mesenchymal transition to pancreatic cancer progression. Cancers 2: 2084-2097, 2010.

11. Shah AN, Summy JM, Zhang J, Park SI, Parikh NU and Gallick GE: Development and characterization of gemcitabineresistant pancreatic tumor cells. Ann Surg Oncol 14: 3629-3637, 2007.

12. Lee HJ, Kim MS, Shin JM, Park TJ, Chung HM and Baek KH: The expression patterns of deubiquitinating enzymes, USP22 and Usp22. Gene Expr Patterns 6: 277-284, 2006.

13. Zhang XY, Pfeiffer HK, Thorne AW and McMahon SB: USP22, an hSAGA subunit and potential cancer stem cell marker, reverses the polycomb-catalyzed ubiquitylation of histone $\mathrm{H} 2 \mathrm{~A}$. Cell Cycle 7: 1522-1524, 2008.

14. Atanassov BS and Dent SY: USP22 regulates cell proliferation by deubiquitinating the transcriptional regulator FBP1. EMBO Rep 12: 924-930, 2011.

15. Park IK1, Qian D, Kiel M, Becker MW, Pihalja M, Weissman IL, Morrison SJ and Clarke MF: Bmi-1 is required for maintenance of adult self-renewing haematopoietic stem cells. Nature 423: 302-305, 2003.

16. Zhang XY, Varthi M, Sykes SM, Phillips C, Warzecha C, Zhu W, Wyce A, Thorne AW, Berger SL and McMahon SB: The putative cancer stem cell marker USP22 is a subunit of the human SAGA complex required for activated transcription and cell-cycle progression. Mol Cell 29: 102-111, 2008.

17. Yang DD, Cui BB, Sun LY, Zheng HQ, Huang Q, Tong JX and Zhang QF: The co-expression of USP22 and BMI-1 may promote cancer progression and predict therapy failure in gastric carcinoma. Cell Biochem Biophys 61: 703-710, 2011.

18. Shook D and Keller R: Mechanisms, mechanics and function of epithelial-mesenchymal transitions in early development. Mech Dev 120: 1351-1383, 2003.

19. Yang $\mathbf{J}$ and Weinberg RA: Epithelial-mesenchymal transition: at the crossroads of development and tumor metastasis. Dev Cell 14: 818-829, 2008.

20. Glinsky GV: Genomic models of metastatic cancer: functional analysis of death-from-cancer signature genes reveals aneuploid, anoikis-resistant, metastasis-enabling phenotype with altered cell cycle control and activated Polycomb Group $(\mathrm{PcG})$ protein chromatin silencing pathway. Cell Cycle 5: 1208-1216, 2006.

21. Lin Z, Yang H, Kong Q, Li J, Lee SM, Gao B, Dong H, Wei J, Song J, Zhang DD and Fang D: USP22 antagonizes p53 transcriptional activation by deubiquitinating Sirtl to suppress cell apoptosis and is required for mouse embryonic development. Mol Cell 46: 484-494, 2012.

22. Bruce B, Khanna G, Ren L, Landberg G, Jirström K, Powell C, Borczuk A, Keller ET, Wojno KJ, Meltzer P, Baird K, McClatchey A, Bretscher A, Hewitt SM and Khanna C: Expression of the cytoskeleton linker protein ezrin in human cancers. Clin Exp Metastasis 24: 69-78, 2007.

23. Hunter KW: Ezrin, a key component in tumor metastasis. Trends Mol Med 10: 201-204, 2004.

24. Fievet BT, Gautreau A, Roy C, Del Maestro L, Mangeat P, Louvard D and Arpin M: Phosphoinositide binding and phosphorylation act sequentially in the activation mechanism of ezrin. J Cell Biol 164: 653-659, 2004.

25. Thompson EW and Williams ED: EMT and MET in carcinoma clinical observations, regulatory pathways and new models. Clin Exp Metastasis 25: 591-592, 2008. 
26. Pujuguet P, Del Maestro L, Gautreau A, Louvard D and Arpin M: Ezrin regulates E-cadherin-dependent adherens junction assembly through Rac1 activation. Mol Biol Cell 14: 2181-2191, 2003.

27. Egeblad M and Werb Z: New functions for the matrix metalloproteinases in cancer progression. Nat Rev Cancer 2: 161-174 2002.

28. Duong TD and Erickson CA: MMP-2 plays an essential role in producing epithelial-mesenchymal transformations in the avian embryo. Dev Dyn 229: 42-53, 2004.

29. Borghaei RC, Rawlings PL Jr, Javadi M and Woloshin J: NF- $\kappa \mathrm{B}$ binds to a polymorphic repressor element in the MMP-3 promoter. Biochem Biophys Res Commun 316: 182-188, 2004.
30. Hanks SK and Polte TR: Signaling through focal adhesion kinase. Bioessays 19: 137-145, 1997.

31. Schaller MD: Cellular functions of FAK kinases: insight into molecular mechanisms and novel functions. J Cell Sci 123: 1007-1013, 2010

32. Chen Y, Wang D, Guo Z, Zhao J, Wu B, Deng H, Zhou T, Xiang H, Gao F, Yu X, Liao J, Ward T, Xia P, Emenari C, Ding X, Thompson W, Ma K, Zhu J, Aikhionbare F, Dou K, Cheng SY and Yao X: Rho kinase phosphorylation promotes ezrin-mediated metastasis in hepatocellular carcinoma. Cancer Res 71: 1721-1729, 2011. 